\title{
Linking Macroscopic Fracture Properties to Single Dislocation Processes
}

Inas Issa ${ }^{1,2}$, Markus Alfreider ${ }^{2}$, Darjan Kozic ${ }^{3}$, Otmar Kolednik ${ }^{1}$, Stefan Sandfeld ${ }^{4}$ and Daniel Kiener ${ }^{2}$

1. Erich Schmid Institute, Austrian Academy of Sciences, Leoben, Austria.

2. Department Materials Physics, Montanuniversität Leoben, Austria.

3. Material Center Leoben, Forschung GmbH, Leoben, Austria.

4. Chair of Micromechanical Materials Modelling, Institute of Mechanics and Fluid Dynamics, TU Bergakademie Freiberg, Germany.

Understanding fracture processes in structural and functional materials is of utmost importance in order to improve material properties and design failure resistant components and systems. The challenge here lies in the fact that, while failure can destroy large structures in an instant, the underlying crack initiation and propagation processes occur on a very local scale.

In order to bridge these scales, we address brittle as well as elasto-plastic fracture processes in a combined scheme utilizing in-situ testing in electron microscopes along with numerical analysis. In fact, we combine miniaturized dynamical fracture testing in scanning electron microscopes (SEM) (e.g. Figure 1) [1,2] with in-situ transmission electron microscopy (TEM) [3]. The former allows to test representative bulk properties and at the same time monitor crack propagation, either directly from the in-situ images or from the continuously measured change in specimen stiffness upon crack propagation (e.g. Figure 2). The latter enables to identify the initial nucleation processes of individual dislocations at different slip systems at a crack tip. These experimental techniques are paired with finite element analysis (FEA) in order to determine valid geometry factors for the SEM experiments and discrete dislocation analysis to address the local stress and strain state at the crack tip and the connected crack driving forces in the in-situ TEM experiments.

This suite of techniques enables for the first time to bridge from macroscopic properties to individual dislocation processes at a crack tip. Notably, this approach is generally applicable to any material that can be exposed to vacuum conditions and tolerates an electron beam for imaging. Moreover, our analysis extends the current state-of-the-art from miniaturized testing of quasi-brittle materials towards the more general and more demanding case of elasto-plastic fracture processes.

To demonstrate the detailed insights achievable in order to understand global fracture processes on a local scale, selected examples are presented for brittle and quasi-brittle fracture processes in ceramic and metallic materials.

References:

[1] R Fritz et al, Measurement 110 (2017), p. 356.

[2] M Alfreider et al, Materials \& Design (2018), submitted.

[3] E Hintsala et al, Experimental Mechanics 55, (2015), p. 1681.

[4] The authors acknowledge funding from the Austrian Science Fund FWF (P25325-N20), the Austrian Research Promotion Agency FFG within the framework of the COMET Funding Programme (IC-MPPE A1.25) and the European Research Council (ERC-2017-CoG 771146). 


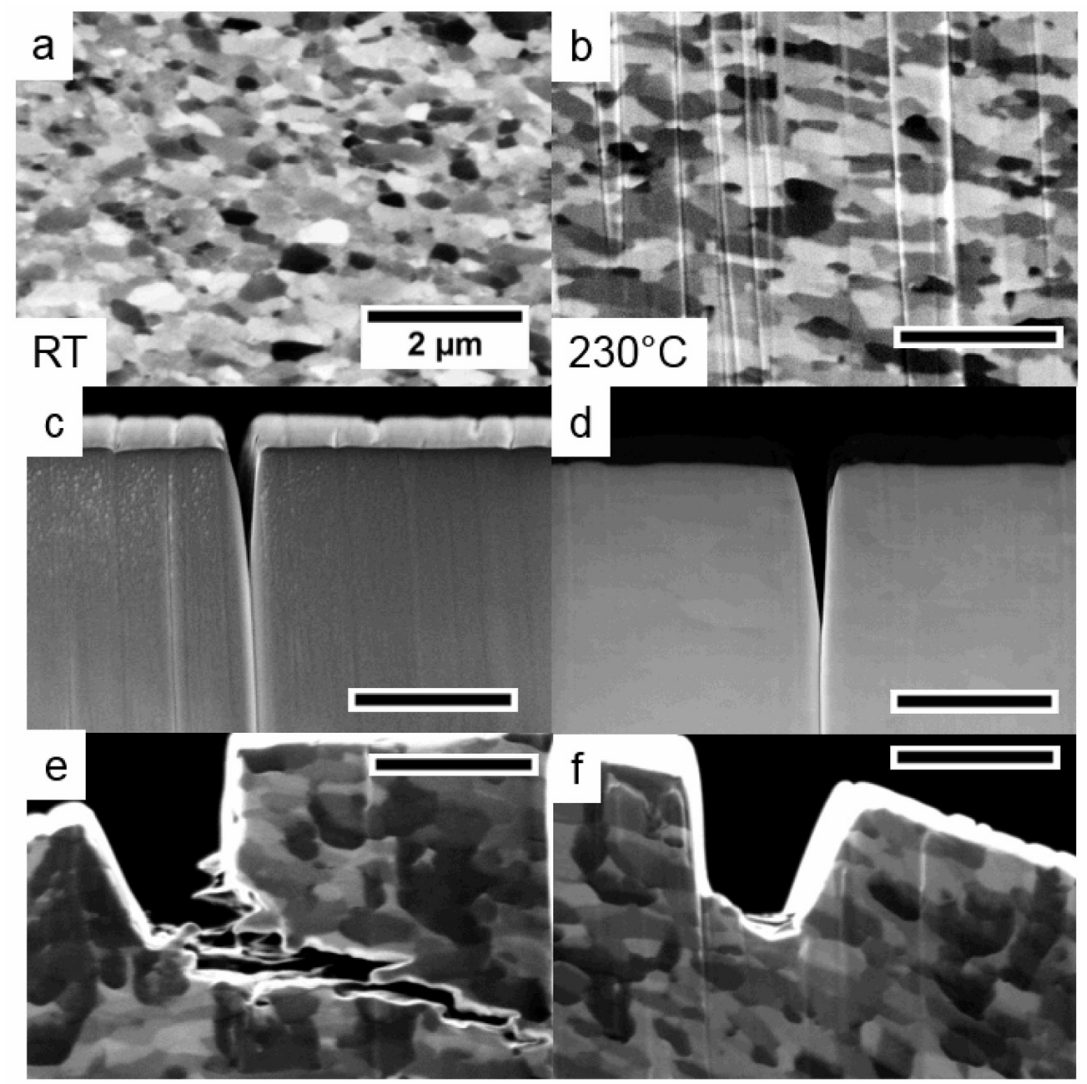

Figure 1. Initial microstructure (top), FIB introduced crack (middle) and final failure mode (bottom) for nanocrystalline $\mathrm{Cr}$ tested in-situ in the SEM at RT (left) and $230^{\circ} \mathrm{C}$ (right), respectively. A change from grain boundary failure to crack blunting is evident. $2 \mu \mathrm{m}$ scale marker applies to all images [1].
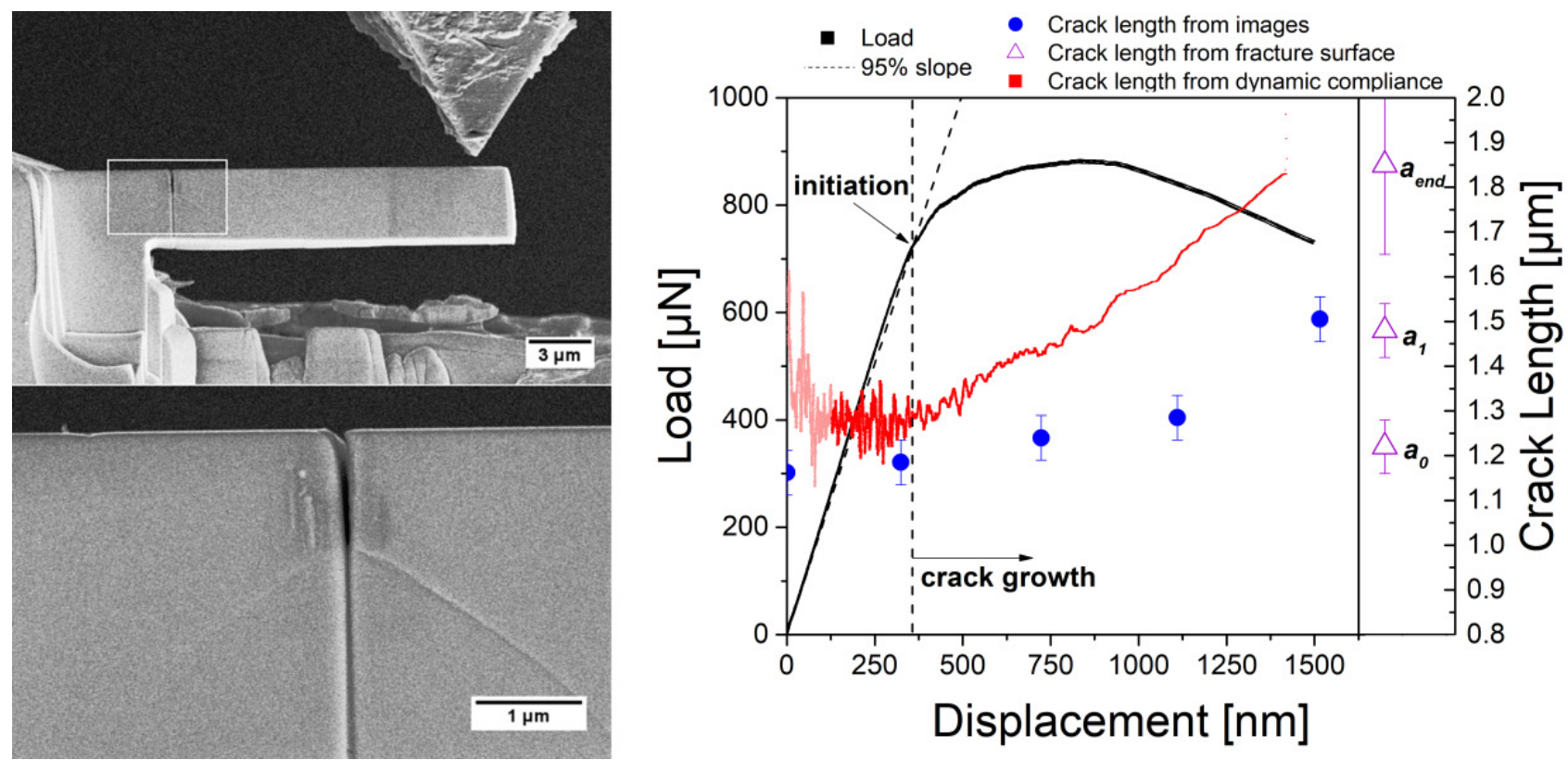

Figure 2. Overview and detail of slip steps emitted from a crack during an in-situ SEM fracture experiment on single crystal tungsten (left), along with related load-deflection and continuous crack extension data (right) [2]. 